\title{
Análisis de la acción del Consejo Para la Transparencia en el proceso de implementación de la política de acceso a la información pública: Un estudio de caso de modernización de la gestión pública
}

Elizabeth Aguilera

Universidad de Chile

\section{Resumen}

El presente estudio analiza el proceso de implementación de la Política de Acceso a la Información Pública (PAIP), con el propósito de identificar las principales fuerzas que influyeron en la instalación y puesta en práctica de esta política pública. A partir del estudio de sus fuerzas impulsoras, se identificaron los factores con mayor influencia y el rol que ellos jugaron en el proceso de implementación de la PAIP Adicionalmente, el análisis de estas fuerzas impulsoras ha contribuido a comprender la estrategia y obstáculos enfrentados por el CPLT para encaminarse a lograr el valor público declarado. Se concluye que las relaciones de poder, la búsqueda de racionalidad y el ajuste organizacional han sido las principales fuerzas que han influido en la implementación de la PAIP.

Palabras clave: Modernización del Estado, Implementación de políticas públicas, transparencia, Derecho de acceso a la información pública.

Analysis of the Action of the Council for Transparency in the Implementation Process of the Policy on Access to Public Information: A Case Study of Public Management Modernization

\begin{abstract}
This study analyzes the process of implementation of the Política de Acceso a la Información Pública (PAIP), in order to identify the main forces that influenced the installation and implementation of this public policy. From the study of its driving forces, the most influential factors and the role they played in the process of implementing the PAIP were identified. In addition, analysis of these driving forces helped to understand the strategy and obstacles faced by the CPLT to help to bring about the public value declared. It is concluded that the relations of power, the pursuit of rationality and organizational adjustment have been the main forces that have influenced the implementation of the PAIP.
\end{abstract}

Keywords: State modernization, public policies implementation, transparency.

\section{Política de Acceso a la Informa- ción Pública ${ }^{1}$}

La Política de Acceso a la Información Pública responde primeramente a una lógica de reforma del

\footnotetext{
${ }^{1}$ Esta investigación fue parcialmente financiada en el marco del proyecto Fondecyt $\mathrm{N}^{\circ} 1120545$ a cargo del profesor y académico de la Universidad de Santiago de Chile Mauricio Olavarría Gambi.

*Dirección de correspondencia [Correspondence
address]:
Elizabeth Aguilera, Universidad de Chile
E-mail: eliaguilera@ug.uchile.cl
}

Estado y modernización de la gestión pública que ha sido desarrollada mediante un proceso gradual e incremental, iniciado a partir de la década de 1990, destinado a mejorar el funcionamiento de los organismos públicos. Ese proceso puso como eje de los cambios a los ciudadanos, fortaleciendo la eficiencia, la eficacia y la calidad de la gestión pública, mediante la instalación de mecanismos que ayudasen a la rendición de cuentas y la transparencia de los actos de la administración del Estado.

El surgimiento de la Política de Acceso a la Información Pública es parte de una preocupación de larga duración por hacer un uso adecuado de los recursos públicos. Esta política es parte de los impulsos del Estado chileno por modernizar la gestión 
pública, mediante el reconocimiento progresivo de la necesidad de hacer efectivo el ejercicio de los derechos ciudadanos. Bajo estos estos antecedentes, nace la Ley $20.285^{2}$ sobre Acceso a la Información Pública, consagrando el derecho de acceso a la información del Estado y a la creación del Consejo para la Transparencia, organismo encargado de implementar la Ley.

La importancia de abordar un estudio como este, viene dada por conocer el proceso de implementación que tiene una política, en términos de como este se adapta bajo el marco que está regido por una ley o reglamento, como es este caso, principalmente dado por cambios que están condicionados por ciertas fuerzas o factores que impactan directamente en dicho proceso.

En este contexto, es relevante entender cuáles fueron los factores o fuerzas que incidieron en la implementación de la Política de Acceso a la Información Pública, así como cuáles fueron las acciones que emprendió el Consejo para la Transparencia para hacer efectiva la Ley 20.285. Es por esta razón, que la presente investigación busca identificar cuáles fueron los principales factores que incidieron en el proceso de implementación de esta política, el rol que esta ha cumplido, así como la importancia que esta ha alcanzado. Por otra parte, se busca comprender cómo estos factores han sido percibidos como impulsores u obstaculizadores hacia el cumplimiento de la política, dando a conocer, de esta forma, las estrategias y modelos de gestión política llevados a cabo para la generación de valor público.

\section{Análisis de implementación a través del enfoque de driving forces do- mains}

En el contexto del proceso de implementación de una política pública, la literatura entrega una vasta información con respecto a los diferentes enfoques que se han llevado hasta el momento, pero sin lugar a dudas no existe un camino correcto per se, sino más bien, es el conjunto de factores históricos que tenga un país, junto con la finalidad con que se generó la política que impactan en el cómo será su implementación, por ello es importante dar conocer el debate de estos enfoques para reconocer sus diferencias y el objetivo que prima en cada uno de ellos.

Producto del debate de las perspectivas más

\footnotetext{
${ }^{2}$ Ley 20.285, sobre Acceso a la Información Pública. Fue publicada el 20 de agosto del 2008 y su reglamento al año siguiente el 13 de abril del 2009.
}

clásicas sobre la implementación de políticas públicas, los esfuerzos se han orientado en torno a sintetizar los enfoques top down y bottom up. Según plantean Hill y Hup (2002), los enfoques mixtos pueden ser utilizados ya que ambos son complementarios, pero el contexto institucional influirá directamente en el enfoque desde el cual se aborde la política pública. Por lo tanto, según plantea Olavarría (2014:4-5) el estudio de la implementación de políticas estaría dado por la "búsqueda de factores (o variables) asociados a un resultado real o previsto de una intervención política".

Según Hasenfeld \& Brock (1991), la implementación de políticas puede ser caracterizada en base a las interacciones que realizan los ejecutores de política dentro de un espacio inter-organizacional. Olavarría (2014:5) señala que "la implementación de políticas puede ser caracterizada como la interacción dinámica de los siguientes dominios de fuerzas motrices: la búsqueda de la racionalidad, la forma política de la organización, la discreción burocrática y la adaptación, las relaciones de poder, y liderazgo y competencia".

Los autores Hasenfeld \& Brock (1991), le llaman fuerzas impulsoras (driving forces domains) y hacen referencia a las explicaciones ofrecidas a partir de los patrones observados al realizar el estudio. Por lo tanto, estas fuerzas pueden ser entendidas también como las dinámicas que prevalecieron durante el proceso de implementación, actuando como impulsos en el momento de llevar adelante la ejecución de la política pública.

La primera de estas fuerzas es la búsqueda de la racionalidad. Esta hace mención a la relación entre los objetivos de la política y los medios asignados para su ejecución. Así, el resultado es la búsqueda por obtener productos, a partir de un proceso planificado y de ejecución racional, siempre que sus ejecutores se comporten de acuerdo al estatuto o mandato político.

En segundo lugar está el ajuste organizacional, el que tiene relación a la capacidad que tienen los implementadores dentro del aparato estatal para desarrollar las estructuras y procesos internos que se ajusten a los patrones institucionales. Según lo planteado por Lawrence y Lorsch (1967), las organizaciones tienden a crear rutinas o procedimientos de trabajo que reflejan la contingencia interna en términos de lo habitual y previsible, en respuesta a los problemas más comunes que suceden a diario en la organización.

La discreción burocrática, se refiere al comportamiento que tienen los burócratas de primera línea (a nivel de calle), en la prestación de servicios. 
Se propone que durante el curso de la ejecución de una política, esta es controlada gradualmente mediante el ejercicio discrecional de los ejecutores o burócratas. Según Weatherley y Lipsky (1977), estos burócratas pueden usar esta discreción para mantener ciertas rutinas organizativas existentes subvirtiendo así, la intención verdadera de la política a ejecutar.

Las relaciones de poder, dan cuenta de cuan determinantes son las relaciones entre los distintos grupos de interés en el destino que tome el proceso de implementación, ya sea, mediante el control de recursos o la negociación, en especial cuando hay que considerar que una política puede alterar intereses de un cierto grupo, como también trastocar valores que sean importante para un segmento de la sociedad. Según señalan Levin \& Ferman (Levin y Ferman, 1985:35) "el núcleo de la política es el conflicto de intereses. Los individuos y las organizaciones, por lo tanto, se resisten a la aplicación de una política o programa, ya que no lo ven como miembros de sus intereses".

Por último, el liderazgo y la competencia, dan cuenta que la aplicación está en función de las cualidades de liderazgo, habilidades interpersonales y la competencia de sus ejecutores (directivos o ejecutores). En este sentido, Levin y Ferman (1985) hacen hincapié en el papel de liderazgo del Ejecutivo como un "fijador" en la reparación y ajuste del proceso de implementación, y como un "doble agente" en la obtención de una acción conjunta entre los distintos intereses.

Este enfoque, reconoce que su aplicación tiene que centrarse en la interacción entre la formulación de políticas, los instrumentos de política, y el o los sistemas organizativos que responden a ello (Elmore, 1987). Las acciones de los sistemas de organización en la ejecución están acotadas y limitadas por los instrumentos de política, pero también reflejan los intereses de la organización (Sabatier, 1987). Por consiguiente, una teoría debe tener en cuenta las características de una política, los factores en las relaciones entre las organizaciones que tienen un interés en dicha política (es decir, la red de la organización), y las costumbre o valores que se establecen a partir de las relaciones dentro de la organización.

Según Hasenfeld \& Brock (1991:456), "son las relaciones de poder el factor predominante en este estudio, sin importar el enfoque que tengan las políticas estudiadas". Como segundo factor a destacar, si bien muy condicionado al entorno, es el de discreción burocrática, que los autores interpretan como otra manifestación de las relaciones de poder, que se hace presente en especial, entre el personal de nivel inferior, al que se ha denominado como ejecutores a nivel de calle.

La racionalidad surge como otra fuerza motriz que los autores del estudio consideran importante, estos plantean que una variable a considerar dentro de este conjunto de trabajos fue el "entorno inter-organizacional en el cual se ejecutaban las políticas", de este modo, el factor de "ajuste organizacional salió presente en los resultados" haciendo mención a los aspectos económicos que estuvieron presentes. De ahí que las tres fuerzas impulsoras: relación de poder (incorporando la discreción burocrática dentro de este factor), la racionalidad técnica y económica como expresión del ajuste organizacional, constituyen las fuerzas predominantes dentro de este conjunto de trabajos.

Los autores Hasenfeld \& Brock (1991) terminan señalando que este enfoque permite apreciar los procesos de implementación, explicando las dinámicas que se producen y el resultado final, es decir, la salida política. Por otra parte, parece más fructífero considerar el proceso de implementación como un aprendizaje organizacional iterativo, de tiempolimitado, y múltiples participaciones de ejecutores como también de otros organismos.

Similar estudio se realizó en Chile utilizando los mismos driving forces domains del estudio de Hasenfeld \& Brock (1991). El estudio "Policy Implementation: Lessons from the chilean policy on $\mathrm{pu}-$ blic management modernization" (Olavarría, 2014), consideró como unidad de análisis la red interorganizacional en que las Políticas de Modernización de la Gestión Pública en Chile se han implementado.

A partir de los resultados obtenidos por Olavarría (2014), se observa que las principales fuerzas que impulsan la implementación de Política de Reforma y Modernización de la GEstión Pública, son el liderazgo y la competencia, el ajuste organizacional de la política, y la racionalidad. Estos resultados "serían consistentes con una perspectiva de arriba hacia abajo de la implementación de políticas, lo que a su vez, sería también coherente con las características tecnocráticas de la política de modernización de la gestión pública" (Olavarría-Gambi, 2014:25).

\section{Una mirada de valor público a partir de la implementación de políticas}

Moore (1995) hace mención a una estructura de razonamiento que ayuda a los gerentes de las empresas públicas a desarrollar marcos de diagnóstico, que permiten a los administradores analizar los en- 
tornos en los que operan, e identificar el tipo de gestores e intervenciones que pueden realizarse para explotar el potencial de las circunstancias en que se encuentran.

Moore (1995) sigue desarrollando esta idea sobre la base de establecer una estrategia, la cual se concibe como un triángulo estratégico:

- Una declaración de la misión o propósito de la organización (fundido en términos de valores públicos importantes)

- Una descripción de las fuentes de apoyo y legitimidad que se utilizarán para satisfacer el compromiso de la sociedad con dicha tarea (gestión hacia arriba, con el fin de obtener del entorno político la legitimidad y apoyo suficiente para su propósito)

- Una explicación de cómo se debe organizar la empresa para alcanzar los objetivos declarados (gestión hacia abajo, intentando mejorar la capacidad de la organización para conseguir los propósitos deseados)

Por lo tanto, Moore (1995) habla de una doble concepción de valor público. En primer lugar el valor público como un concepto que define un fin último de la acción que pueden llevar adelante los gerentes, al hacer uso de los recursos de propiedad estatal (autoridad y dinero). Y en segundo lugar, el valor público como lo que se señaló en párrafos anteriores, un sistema de razonamiento práctico, del cual los gestores pueden invocar para definir y ejecutar el mandato que les fue encomendado.

El uso del triángulo estratégico, está asociado al desarrollo estratégico por parte de los gestores públicos para la creación de valor, del cual según plantea Moore (1998), el directivo público debe saber alinear la estrategia de modo tal que para los demás (supervisores, clientes y beneficiarios) les sea valiosa en términos sustantivos y posea un bajo costo en términos monetarios y de autoridad. Esta idea corresponde a la parte superior del triángulo en donde el público debe autorizar al organismo a gastar los recursos de modo legítimo. Esta tarea ha implicado por parte de los gestores, una tendencia a centrarse en la obtención de fondos y la demostración de la rendición de cuentas hacia arriba a los ministros, en lugar de una rendición de cuentas más dirigida a los actores locales o al público.

En cuanto a la medición del impacto que tengan las intervenciones con el objeto de crear valor, los organismos públicos muchas veces se enfrentan a una gran diversidad de objetivos, y por tanto, indicadores que no sólo vienen de su misma organización, sino también de otros organismos, ya sea producto de acuerdos de servicio, objetivos de productividad y eficiencia o rendimientos clave, indicadores, evaluaciones, inspecciones y así sucesivamente. En este sentido, Horner \& Hutton (2011) señalan que hay una tendencia a esperar que el valor público puede proporcionar una alternativa superior a todos estos enfoques, pero no se debe olvidar que el concepto de valor público depende del objetivo de la política y de los gestores a cargo, lo que implica que, por sí mismo, tiene sus limitaciones.

La teoría de valor público contribuiría a que el administrador o gerente público, utilice la gestión como una herramienta en la cual implica trabajar en red, generando alianzas con diversas organizaciones, tanto internas (entiéndase con los mismos empleados), así también con el público para ir desarrollando una entrega de servicio más eficaz y una mayor contribución al bienestar social. Equilibrar las necesidades y demandas tanto de los consumidores individuales y el público en general, es algo que pocos directivos de cualquier sector tienen experiencia de hacer. Esta es el área fundamental de hoy para la gestión y desarrollo organizacional.

También los resultados de Hasenfeld \& Brock (1991), hacen hincapié en cuán importante son las relaciones inter-organizacionales y que están presentes cuando se habla de relaciones de poder, porque el gestor puede depender de la implementación de otros actores o agencias para el logro de los resultados según Moore (1995).

También Klijn (2007) plantea que, desde una perspectiva de red o entre organizaciones, la entrega de un servicio se vuelve compleja porque los resultados no son inmediatos y el gestor público tiene que coordinar con cuidado y aprender a gestionar.

La apelación al concepto de valor público le otorga sentido a los objetivos estratégicos, pues permite realizar los ajustes organizacionales para lograr los objetivos y metas planteados por la organización. En palabras de Moore (1995), se puede considerar importante medir el proceso pero en términos de acciones para generar valor público. De este modo se construye un valor público scorecard, como una forma alternativa de medir el rendimiento sin fines de lucro y como un medio de implementar una estrategia de valor público.

Esta orientación implica que los gestores o directivos públicos orienten su planificación estratégica a los elementos de la cadena de valor. Los organismos públicos presentan una dificultad en la práctica que debe ser considerada, porque generalmente todo el valor se encuentra al final de la cadena, en 
los procesos de producción donde se entrega el producto final, en vez de la parte de "arriba" en donde la organización reúne los recursos para obtener los objetivos.

\section{Metodología}

Esta investigación tiene carácter exploratorio (Yin, 1994). La unidad de análisis de este estudio es la red de colaboración en el cual la PAIP se ha implementado . Los datos provienen de tres fuentes: entrevistas, documentos oficiales y prensa de la época. Se llevaron a cabo entrevistas a una muestra intencionada de once personas que han desempeñado roles relevantes en la puesta en funcionamiento de la PAIP, en la que se pudo obtener información clave del proceso. El universo de entrevistados fue compuesto por altos funcionarios públicos, como también directivos de segunda línea y representantes de ONGs. Al ser una muestra intencionada, ayudó a escoger testimonios e información que nutriera las fuerzas o factores pertinentes (descritos anteriormente) categorías analíticas pertinentes y evitar reiteración o duplicación de información, mismo método aplicado en el estudio de Olavarría (2014).

Con el fin de identificar las principales fuerzas impulsoras en el proceso de implementación de la Política de Acceso a la Información Pública, las entrevistas incluyeron preguntas abiertas sobre estrategias abordadas; presupuesto asignado; conceptos y objetivos que primaron a la hora de llevar adelante la implementación, tanto de los factores impulsores como obstaculizadores enfrentados; los actores relevantes y las decisiones tomadas. Las entrevistas se realizaron durante el mes de diciembre de 2013, agosto y septiembre de 2014, en función de la disponibilidad de los entrevistados.

Las entrevistas fueron grabadas, transcritas y después analizadas mediante el método de análisis de contenido (Bardin, 2002). El análisis de las entrevistas permitió identificar factores que influenciaron el proceso de implementación. Estas sentencias fueron agrupadas en relación al papel desempeñado por el entrevistado en este proceso, para fines de este estudio se crearon 3 categorías: Altos Funcionarios, quienes son consejeros, presidente y expresidentes del Consejo para la Transparencia; Gestores públicos, compuesta por directivos de segundo y tercer nivel jerárquico dentro de la administración pública y Otros, formados por personas relacionadas con el tema pero no miembros del Estado (ONG) (altos funcionarios, gestores públicos y otros ). Además de lo anterior, se llevó a cabo un análisis acerca de la idea de valor público que primó durante los primeros cinco años del proceso de instalación de la ley por parte del Consejo.

Con el fin de revisar elementos del contexto, conflicto e información complementaria de los hechos y circunstancias a lo largo de la implementación de la PAIP, se analizaron documentos de prensa tales como: "El Mercurio", "La Tercera" y "La Nación" publicados durante el período estudiado.

Adicionalmente, se revisaron documentos oficiales, en su mayoría estudios realizados por el mismo organismo autónomo, con el objeto de identificar las líneas programáticas de esta política y como estas se implementaron. También se analizaron estudios de satisfacción al cliente, información institucional y reportes estadísticos emitidos, principalmente, por el Consejo para la Transparencia.

Además de lo anterior, se examinó literatura académica, documentos de expertos e informes, con el objetivo de recoger antecedentes conceptuales que permitan caracterizar el proceso de implementación de esta política pública.

\section{Resultados}

A modo general, el cuadro 1 muestra que los entrevistados identifican el factor de las relaciones de poder como la principal fuerza en la implementación de esta política, seguido de la racionalidad; ajuste organizacional; discreción burocrática y adaptación; y liderazgo y competencia . Esta última parece ser menos importante que las otras cuatro fuerzas, sin embargo, tanto altos funcionarios, gestores públicos como otros entrevistados, entre ellos representantes de ONG y otras entidades públicas, perciben unánimemente que un factor crucial a la hora de llevar adelante la instalación de la política de acceso a la información pública son las relaciones de poder. No obstante, el único grupo que presentó diferencias con respecto a las influencias de las fuerzas, fue el grupo de "otros", al manifestar que la segunda fuerza dominante correspondía a: discreción burocrática y adaptación, seguido por racionalidad.

\section{Análisis de fuerzas dominantes duran- te el proceso de implementación según grupo de entrevistados}

\section{Altos Funcionarios}

Los altos funcionarios, por estar más cercanos a la arena donde se discute el curso de instalación de 
Cuadro 1: Importancia relativa a partir de las fuerzas dominantes

\begin{tabular}{|c|c|c|c|c|c|c|c|c|}
\hline \multirow{3}{*}{ Fuerzas dominantes } & \multicolumn{6}{|c|}{ Rol del entrevistado } & \multirow{2}{*}{\multicolumn{2}{|c|}{ Total }} \\
\hline & \multicolumn{2}{|c|}{ Altos funcionarios público } & \multicolumn{2}{|c|}{ Gestores públicos } & \multicolumn{2}{|c|}{ Otros } & & \\
\hline & $\mathrm{N}^{\circ}$ Frases & peso & $\mathrm{N}^{\circ}$ Frases & peso & $\mathrm{N}^{\circ}$ Frases & peso & $\mathrm{N}^{\circ}$ Frases & peso \\
\hline Racionalidad & 46 & 0,25 & 96 & 0,26 & 7 & 0,16 & 149 & 0,25 \\
\hline Ajuste organizacional de la política & 29 & 0,16 & 78 & 0,21 & 6 & 0,14 & 113 & 0,19 \\
\hline Discreción burocrática y adaptación & 32 & 0,17 & 47 & 0,13 & 8 & 0,19 & 87 & 0,15 \\
\hline Relaciones de poder & 59 & 0,32 & 99 & 0,27 & 21 & 0,49 & 179 & 0,30 \\
\hline Liderazgo y competencia & 18 & 0,10 & 46 & 0,13 & 1 & 0,02 & 65 & 0,11 \\
\hline Total & 184 & 1,00 & 366 & 1,00 & 43 & 1,00 & 593 & 1,00 \\
\hline
\end{tabular}

Fuente: elaboración propia, basado en el estudio de Olavarría (2014)

la política pública, centran su atención en las consecuencias que puede traer tomar ciertas decisiones correspondientes al espacio institucional: límites en funciones que adquiera el Consejo para la Transparencia, la construcción de alianzas, estrategias y negociaciones con el objeto de aliviar las tensiones suscitadas producto de la pugna de ideas y objetivos que primen en la implementación de la política. Los resultados mostrados en la Ilustración 1, indican como la fuerza principal en este grupo las relaciones de poder. Se destaca dentro de los entrevistados el conflicto por la asignación presupuestaria y la administración del portal de transparencia.

El Consejo está permanentemente produciendo alianzas con el mundo político para respaldar sus medidas e ir ganando aliados que impulsen cambios a favor de ir expandiendo su espacio institucional, como también, reconocimiento por parte de otros organismos que han sido renuentes a aceptar nuevas obligaciones producto de la ley 20.285 .

Sin lugar a duda, el presupuesto marcó un inicio complicado en este proceso de instalación visto por este grupo de altos funcionarios, lo que motivó a que ideas que en un comienzo tenían en mente, como una gran campaña publicitaria, se postergara.

Algo similar ocurre con la administración del Portal de Transparencia, según explica un consejero "nosotros no teníamos los recursos para poder construir ese software, entonces fue una tensión permanente para poder lograr aquellos recursos adicionales para eso". Este problema no es solamente del Consejo, también para que otros organismos cumplan la ley 20.285 necesitan de recursos, por lo tanto, la disparidad entre las municipalidades afecta el cumplimiento y penetración de la PAIP. Casos como estos hicieron más lento el proceso de instalación del Consejo y sus funciones, haciendo que el problema se concentrara en la falta presupuestaria como la principal respuesta a los inconvenientes en un inicio del proceso de instalación y por tanto el funcionamiento de la ley.

Por lo tanto, la instalación del Consejo como organismo en términos de equipos, personal y lugar físico es un tema, y otro es el proceso que genera este organismo para irradiar la política hacia otros organismos obligados por la ley 20.285.

Para hacer que los conflictos y tensiones no mermaran las iniciativas de instalación y el cumplimiento de las obligaciones que plantea la ley 20.285 hacia el Consejo, se diseñaron estrategias y alianzas con el objeto de ir logrando acuerdos que ayudasen a que entrara en ejercicio la ley, y también el Consejo como organismo garante. En esta materia, comenta un consejero: "para hacer de esto una política transversal, para que pareciera una política de Estado y no solamente de gobierno, se considera políticamente pertinente hacer una indicación sustitutiva sobre este proyecto que tenía esta aura de transversalidad impulsada por los senadores Gazmuri, socialista, y Larraín, de la UDI". Dicha indicación sustitutiva fue tomada en consideración por el gobierno de la Presidenta Bachelet, lo que dio al Consejo y a la Ley un trámite expedito evitando discusiones más complejas y agilizar el acceso a la información pública por parte de los ciudadanos.

La estrategia comunicacional utilizada por el Consejo buscó aumentar la cobertura de la aplicación de la ley, invitando a otras instituciones prestigiosas a participar, mediante el establecimiento de convenios. Otra medida llevada a cabo con este mismo fin, fue la colaboración por medio de capacitaciones dentro del Estado. De esta manera, se realizaron reuniones con la Contraloría, Poder Judicial, municipalidades y Congreso; un trabajo de persuasión importante y necesario. Cabe destacar que todas estas estrategias están impregnadas de un fuerte componente de racionalidad.

El Consejo estableció metas y objetivos, con el fin de operacionalizar las obligaciones y atributos que les planteaba la ley 20.285. Como afirma un entrevistado, lo primero que se trazaron para lograr como Consejo fue proponer normas para aumentar la transparencia. Por lo tanto, un primer desafío de este grupo fue entender lo que implicaba cumplir la ley y dotarse de recursos para luego armar las tareas que eran competencia del Consejo y que estaban señaladas en la ley.

La tercera fuerza presente en este proceso de im- 
Figura 1: Composición de las fuerzas para el grupo de altos funcionarios

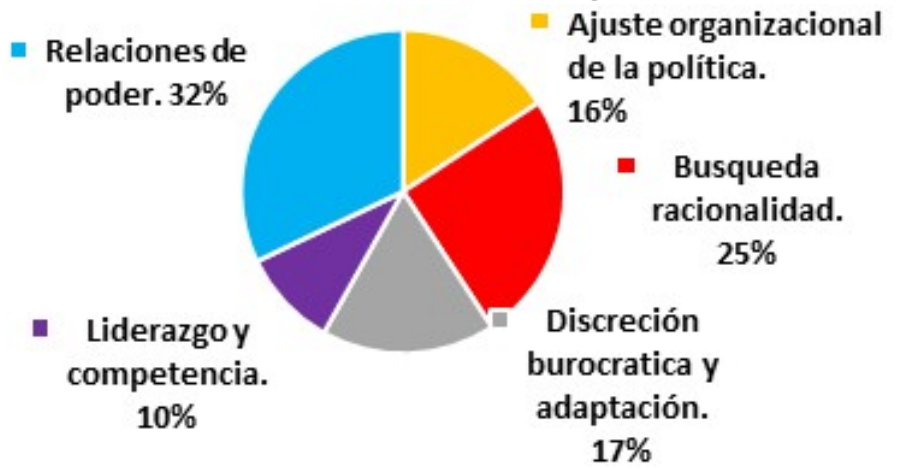

Fuente: Elaboración propia a partir de entrevistas

plementación es la discreción burocrática, la que da cuenta del comportamiento real de los funcionarios. El Consejo se vio en la necesidad de, antes de fiscalizar la aplicación de la ley, enseñarles a los servicios públicos a conocerla y aplicarla. Esto se vio complicado, dado que como comenta un entrevistado: "no hay una cultura de mantener la información sistematizada, de ningún tipo" No existía registro, por lo tanto, la respuesta a la ciudadanía era negar la existencia de información.

En cuanto al ajuste organizacional, entendiendo por este como los esfuerzos por construir y dotar de estructura y procesos al interior del Consejo, este se fue percatando en el transcurso de la aplicación, de las diferencias entre organismos públicos y como esta ley les impactaba en términos de dotación de recursos para hacer cumplir efectivamente con todo lo que exigía la ley 20.285. Un alto funcionario al respecto relata: "Esta ley significa un costo para los organismos de la administración del Estado. Para muchos funcionarios y autoridades es un costo que duele mucho más, porque te distrae personas y recursos de otras actividades", así también en términos generales un entrevistado comenta "los organismos públicos en lo que se refiere a transparencia activa, no tenían ninguna práctica ni costumbre de tener información, no solamente publicada, sino que tampoco al interior del organismo".

En base a lo anterior, el Consejo dispuso de diversas herramientas para apoyar a los organismos. Esto generó que el Consejo fuera adaptando su estructura, mediante la creación de unidades en respuesta a la contingencia y a los problemas que suceden a diario en la implementación. Por medio de las solicitudes que hacen los ciudadanos, el Consejo puede percatarse de los ajustes que debe hacer en la manera de organizarse.

El liderazgo y competencia, es calificada con menor influencia que las otras fuerzas en lo que respecta al impacto en el proceso de instalación de la
PAIP desde la mirada de este grupo de entrevistados, posiblemente por el diseño institucional que rige al Consejo, que responde a un gobierno corporativo, en donde no existe una solo figura o actor protagónico que comande el curso de instalación. El ajuste organizacional de la política generó que el liderazgo o competencia fuesen menos dominantes en el transcurso de implementación de la PAIP y que por tanto el liderazgo no fuere preponderante para concretar el modo en que se iba a llevar la política adelante.

\section{Gestores Públicos}

Los gestores públicos, por su parte, coinciden con los altos funcionarios en señalar cuáles son los tres factores o fuerzas dominantes en este proceso de implementación, pero las razones son distintas en algunos casos, posiblemente porque ellos ejecutan la política diariamente y están más cercanos al cliente final, al que accede a reclamar el derecho de acceso.

Un elemento clave que este grupo destaca dentro de las relaciones de poder es el conflicto emanado producto del presupuesto asignado. Otro conflicto que este grupo destaca, tiene relación con la administración del Portal de transparencia. Este portal estaba en un comienzo en manos del Ministerio Secretaría General de la Presidencia, administrado por la Comisión de Probidad y Transparencia. Actualmente el portal está en manos del Consejo, pero esta administración, la ve condicionada a una voluntad política, lo que se refleja a partir de lo que menciona un entrevistado "Hoy día el portal de transparencia se soporta de la buena voluntad de los municipios y la disposición política del gobierno, pero nada obliga a un alcalde a estar conectado al portal", además la gestión de redes que hace el Consejo con otros organismos y actores del mundo político permite que el portal se mantenga operativo. 


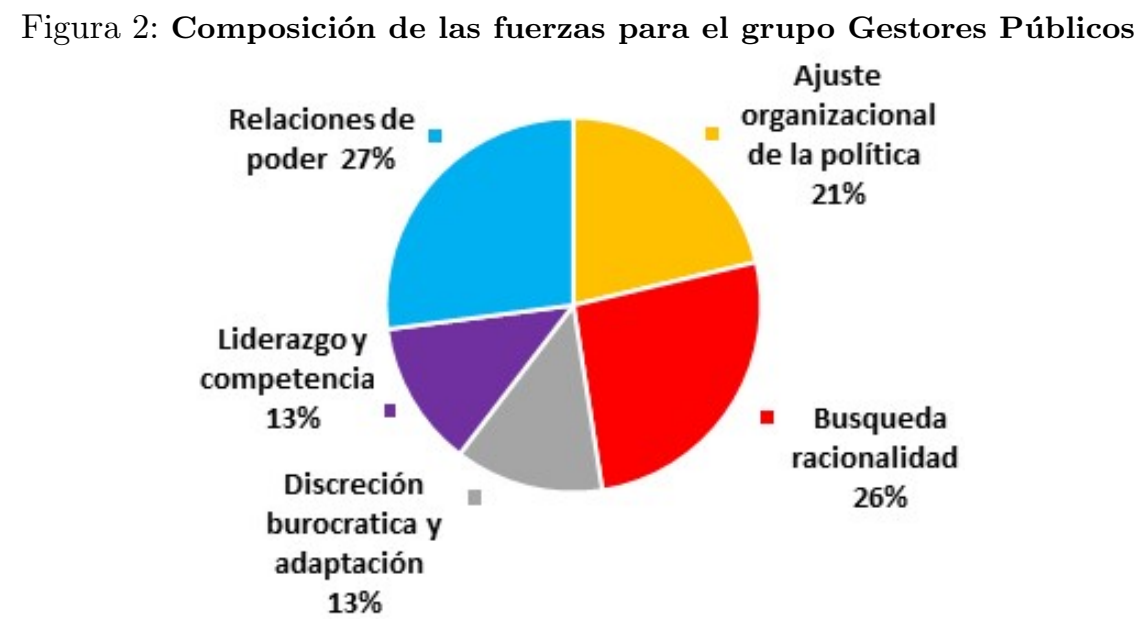

Fuente: Elaboración propia a partir de entrevistas

Producto de estas tensiones, este grupo da a conocer la existencia de estrategias y alianzas, con el objeto de ir calmando los ánimos y darle trazabilidad concreta a las acciones del Consejo. Con la intención de ir ganando espacio en la aplicación de la ley, un entrevistado comenta cuán importante es entender que el "derecho de acceso a la información en la práctica no lo hace el Consejo ni los ciudadanos, lo hacen los funcionarios, por lo tanto tenemos que instalar una alianza con los funcionarios, eso significó abrir una línea de capacitación súper potente". Comprendido lo anterior, este grupo, entiende que las estrategias deben ir al servicio de los organismos públicos, con el fin de ayudarles a cumplir las obligaciones que establece la ley 20.285.

En esta línea, y muy de la mano de esta fuerza, se encuentra la búsqueda de racionalidad. Este grupo percibe que la racionalidad tiene una influencia en la instalación de la PAIP, la cual se manifiesta como señala un directivo "Hay un momento clave a mi juicio que también tiene que ver con hacer un primer levantamiento, partir de la planificación estratégica, cuáles son los procesos que debieran operar dentro del Consejo. Se hace un mapa de proceso, levantamiento de procesos del Consejo". Otro antecedente que se recoge de las entrevistas a este grupo, es la orientación al ciudadano, que se plantea como un objetivo estratégico y que debe por tanto permear todo proceso que se lleve a cabo por parte del Consejo.

El ajuste organizacional aparece asociado al diseño de la estructura y la institucionalización. En ese sentido, un entrevistado revela
El Consejo partió modelando, modeló todo. Hizo sus declaraciones estratégicas fundamentales, modeló los procesos del Consejo, dentro de los procesos del Consejo identificó cuál era la estructura orgánica más apropiada, porque esto partió con una estructura orgánica que no es la que tenemos hoy día, porque de alguna manera fuimos definiendo una estructura, el organigrama, derechamente, en función de estos objetivos estratégicos, en función de los procesos

Pero justamente este proceso de ajuste de procedimientos con la contingencia interna y externa, no estuvo ajena de conflictos al interior del Consejo, pudiendo que parte de las acciones que se llevaron a cabo estuviesen marcadas con una cierta visión de hacer las cosas con predominio frente a otros actores dentro del organismo. Ese temor que se expresa a partir de las entrevistas, puede ser producto de la reacción de este grupo de jefes de segundo nivel en el Consejo, en donde al tener más contacto con el usuario, diseñan y crean controles para hacer frente a las incertidumbres y las presiones de la contingencia.

Este grupo coloca mucho énfasis en las unidades que se crearon para hacer funcionar el Consejo, como señala un entrevistado "tenemos un negocio de regulación, porque podemos promover instrucciones generales y también diseñar perfeccionamientos normativos, también tenemos el negocio de la promoción y capacitación, también tenemos el negocio de la atención de clientes, tenemos el negocio de la fiscalización y el negocio de la solución de casos".

Según estos testimonios, parece ser que el ajuste organizacional de la política está muy vinculado al aspecto racional por el uso de la herramienta de Balance Scorecard y las Normas ISO, posiblemente con el objeto de lograr y medir eficacia en los 
esfuerzos por implementar la PAIP.

Tanto el liderazgo y competencia como la discreción burocrática y adaptación, son fuerzas en menor medida reconocibles por parte de este grupo de gestores públicos. Sin embargo, respecto a la discreción burocrática, se reconoce al interior del consejo que hay por parte de los funcionarios de la administración pública, un conflicto en la valorización, como señala un entrevistado, "una dicotomía en esa valoración, una valoración genérica positiva y una valoración práctica, operativa que es menos positiva y creo que también dificulta el hecho de ser eficiente en el ejercicio del derecho y la aplicación de la política". Por lo tanto la PAIP, por parte del Consejo, ha sido implementada de modo gradual, entendiendo la disparidad de recursos en los organismos afectos a la ley 20.285 .

Un elemento clave que reconoce este grupo de entrevistados, es la capacidad que tienen los funcionarios para desarmar una política, por eso es tan importante la capacitación y las relaciones de poder en términos de establecer negociaciones.

En relación al liderazgo y competencias, tiene una dimensión que es interna dentro del Consejo, donde como comenta un entrevistado "el liderazgo de los presidentes en particular no fue crítico y no ha sido tan crítico como uno se lo imaginaría, el liderazgo se ha dado más bien en el equipo técnico político y el que ha conducido la política pública de verdad desde dentro" y por otro lado está el impulso y compromiso de los otros organismos con la ley, en ese sentido un directivo señala lo siguiente "el liderazgo del alcalde condiciona mucho el equipo de gestión que hay, el nivel de formación que tienen, cosas de ese tipo".

\section{ONGs y otras entidades}

Con respecto al grupo compuesto por ONGs y otras entidades, estos perciben, perciben la implementación de la PAIP, marcada fuertemente por las relaciones de poder, muy por sobre las otras fuerzas dominantes. Como dan a conocer los relatos de los entrevistados, las tensiones están relacionadas con episodios que también fueron dados a conocer en los periódicos en Chile, que son el conflicto del presupuesto asignado al Consejo en sus inicios, el tema con SEGPRES y la tensión con la Contraloría General de la República (CGR), principalmente en la etapa de discusión. Según Olavarría (2012:37) "el Contralor General de la República reaccionó a la indicación del gobierno de incluir a la Contraloría entre los órganos de aplicación de la ley" por ser las normas de transparencia parte de las atribuciones de la misma CGR, presentando entonces duplici- dades con el Consejo en cuanto a la capacidad de fiscalización. Este último hecho con el tiempo se ha ido convirtiendo en una alianza.

Posiblemente, esta percepción de parte de este grupo de entrevistados se debe a que parte de la puesta en marcha del Consejo tuvo retrasos, se tuvo que modificar algunas tareas en favor de priorizar otros aspectos, en vista del presupuesto que fue motivo de disputas, por lo tanto, este grupo, que en parte son clientes o socios estratégicos, en un principio percibieron lentitud en la puesta en marcha de la implementación de la PAIP.

De igual modo, este grupo reconoce que a pesar de las tensiones dadas a conocer, el Consejo ha tenido un acercamiento con los demás órganos con el objeto de hacerlos parte de esta implementación del modo más fácil y menos costoso para los organismos.

Desde la mirada de la ONG, las relaciones de poder se presentan cuando se hace mención al espacio institucional a ocupar por parte del Consejo. El hecho de contar con el portal, avala al Consejo como autoridad en el tema y además le da visibilidad frente al resto de los organismos públicos. Pero aun cuando este grupo reconoce el esfuerzo por parte del Consejo en ir posicionándose también entienden como afirma un entrevistado "ha ocurrido una tensión importante entre el Consejo y la Secretaria General de la Presidencia, porque obviamente el Consejo le pone un peso al Estado".

Otro aspecto que este grupo de entrevistados describe como un problema es la manera en la cual se nombra a los consejeros, mecanismo que afecta a la autonomía del Consejo para la Transparencia. Bajo ese contexto, la ley 20.285 señala: "La dirección y administración superior del Consejo corresponderá a un Consejo Directivo integrado por cuatro consejeros designados por el Presidente de la República, previo acuerdo del Senado, adoptado por los dos tercios de sus miembros en ejercicio. El Presidente hará la proposición en un solo acto y el Senado deberá pronunciarse respecto de la propuesta como una unidad." 3 , por lo tanto, este proceso denota una falta de transparencia en cuanto a quienes conformarán este organismo, si los candidatos poseen o no las aptitudes para asumir un cargo de esta índole, así también, como lo deja ver un entrevistado en la siguiente afirmación "el consejero debe ser una persona capacitada, idónea técnicamente, autónoma, que haya tenido un trato parcial y que entienda también la privacidad y la protección de datos".

Dentro de las fuerzas que destacan, pero en mu-

${ }^{3}$ CHILE. Ley 20.285 , art. 36 . 
Figura 3: Composición de las fuerzas para el grupo ONGs y otras entidades

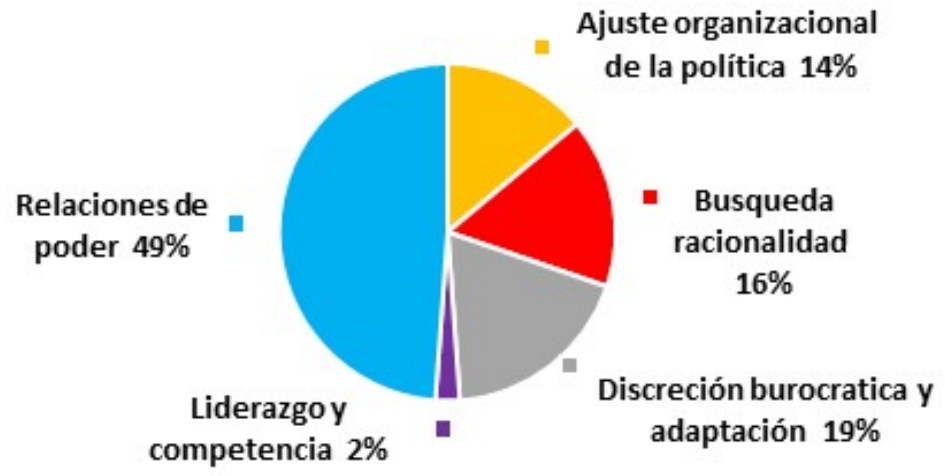

Fuente: Elaboración propia a partir de entrevistas

cha menor cuantía que las relaciones de poder, está el Ajuste organizacional de la política, posiblemente porque la ley 20.285, cambió la relación del usuario con el servicio público, por lo tanto, muchos organismos del Estado tuvieron que verse en la obligación de diseñar procesos en favor de responder según los plazos que la ley establece.

En cuanto a los demás factores, y la influencia de estos en la implementación de la política, es muy leve. Se destaca el carácter autónomo que tiene el Consejo. Otro aspecto que destaca un entrevistado es que el Consejo acerca el ejercicio de la transparencia al ciudadano común.

Con respecto al nivel de adaptación burocrática, este grupo destaca que son pocos los organismos que antes de la aplicación de la ley, tenían incorporados procesos y cultura a disposición de los ciudadanos, a diferencia de ello se destaca la Contraloría. Eso da a entender la disputa de este organismo con el Consejo en aspectos de áreas de competencia.

Un aspecto del factor del liderazgo y competencia, tiene relación con la capacidad de empoderamiento por parte del Jefe o Director al momento de implementar la PAIP. Se destaca en las entrevistas la figura de "la presidenta Bachelet le pone impulso a la agenda de probidad y convoca a una comisión, y esa comisión al final se convence que era necesario hacer un Consejo para la Transparencia", aquello sumó un respaldo importante para que el Consejo, ya en su proceso de implementación, viera en la figura de la Presidenta un respaldo a sus acciones.

Es relevante el interés de Directivos o Subsecretarios en el tema, pues este hace que en la práctica, el servicio genere los procesos y responda a las solicitudes de la ciudadanía en los tiempos que establece la ley.

\section{Análisis de la generación de valor público por parte del Consejo para la Transparencia.}

Los "altos funcionarios", según los testimonios recopilados, asocian la idea de valor público con las funciones principales que tiene el Consejo para la Transparencia actualmente, las cuales son: fiscalización de la transparencia activa y solicitudes de amparos, que se dan a partir de la negación de información a las personas al momento de solicitarla a órganos del Estado. Por ende, el valor público está dado por "aumentar el conocimiento de la ciudadanía sobre la ley de acceso a la información pública, yo creo que es lo más importante, o sea, en la medida que la ciudadanía reclame su derecho, los órganos públicos van a estar más, por decirlo así, presionados para mejorar sus índices de transparencia".

Por lo tanto, bajo la idea de resguardar el valor público que ellos manifestaban en el transcurso de la implementación de la PAIP, se hacía necesario cultivar apoyos, alianzas y/o negociaciones con los demás actores, en vista de que las relaciones de poder marcan fuertemente el proceso de instalación de esta política.

De este modo, el gestor o consejero, en este caso, cumple la función de legitimar las acciones que hace este órgano autónomo a medida que se fortalecen sus lazos con los demás órganos de la administración pública, gana en espacio la institución y de este modo cumplir la idea de valor público que se trazó.

Ahora bien, parte del triángulo planteado por Moore (1998) a modo de estrategia, se puede apreciar a partir de este grupo, pues según los testimonios se pone mucha atención en el tema de posicionamiento del Consejo para la Transparencia dentro del espacio inter-organizacional, con el objeto de lograr autorización en términos de uso de los re- 
cursos, legitimando sus acciones y, por lo tanto, el despliegue mismo de la estrategia.

En cuanto a las entrevistas del grupo de "gestores públicos", se puede constatar que el valor público que se desea alcanzar se refleja claramente a partir del testimonio de un entrevistado: "el valor que estamos agregando acá, es que la información pública sirve para el desarrollo biográfico de los ciudadanos, es decir, es una herramienta de empoderamiento ciudadano, de mejoras de las instituciones y de mejora de la calidad de la democracia" por ello la preocupación de los directivos de establecer controles y procesos que midan el nivel de cumplimento, pues como un entrevistado señala: "el negocio principal del Consejo es para los ciudadanos, a su exigencia de recibir información".

Esta declaración no es contradictoria con lo que se plantea por parte de los altos funcionarios del Consejo para la Transparencia, de hecho suma y profundiza aún más en la idea de que al aumentar el conocimiento de la LAIP, el ciudadano podrá empoderarse frente a las acciones del Estado, y por consiguiente, traería una mayor eficiencia a los organismos públicos, cada vez que se debe responder a las exigencias planteadas por la sociedad al funcionamiento de los servicios públicos. Es por ello que durante el análisis de los factores dominantes del proceso de implementación de la PAIP por parte de los gestores públicos, la búsqueda de la racionalidad está presente fuertemente en los testimonios.

Por otra parte, conjuntamente con la ejecución racional, se encuentra el ajuste organizacional de la política que este grupo imprime al momento de instalar el diseño de procesos. Así menciona un entrevistado "hay un relato entre cada una de ellas, la regulación insume a la promoción, la promoción insume a la atención de consultas, la promoción a su vez y la regulación insume a la fiscalización, la fiscalización en cuanto a su brecha ya llega al proceso de solución de conflictos y lo que veamos en el proceso de solución de conflictos igual que lo que veamos en la fiscalización".

Las entrevistas del grupo llamado "otros", dan a conocer que la idea de valor público se concentra sobre el concepto de cultura de transparencia, y que tiene directa relación con las solicitudes de información por parte de la sociedad, como señala un entrevistado: "ese órgano autónomo al final del día... tiene la capacidad de crear una cultura de la transparencia".

Lo anterior tiene relación a la parte del triángulo estratégico, en la cual se declara la misión o propósito de la organización. A ese punto hace mención este grupo, y esto se vincula directamente al por qué las relaciones de poder se perciben tan fuertemente en este conjunto de testimonios, pues apela directamente a los valores que son importantes para la sociedad, el reconocimiento de un bien o servicio. Pero aquello, como se ha mencionado, no ha estado ajeno a problemáticas que han dilatado que este valor sea de reconocimiento masivo, similar objetivo que buscan los altos funcionarios.

A modo general, las entrevistas de los diferentes grupos, dan la impresión de una comprensión homogénea de la idea de valor público que se quiere transmitir con objeto del proceso de implementación de la PAIP.

Esta idea homogénea está dada principalmente por ciertas fuerzas que tuvieron una marcada presencia en cada uno de los grupos de entrevistados. Comenzando con la búsqueda de racionalidad, posiblemente porque la PAIP es parte de un esfuerzo por dotar de mayor efectividad en el funcionamiento de los organismos estatales, dentro del marco del proceso de modernización de la gestión pública en Chile surgido a partir de 1990 en adelante. Por lo tanto, esta idea de efectividad y eficiencia en el uso de los recursos, está presente en el modo de planificar la instalación del Consejo y sus funciones.

Otra fuerza presente de modo transversal es el ajuste organizacional de la política. Este factor está muy enlazado con el anterior, ya que la idea de aumentar el conocimiento y empoderar a los ciudadanos para generar una cultura de transparencia, requiere también de un esfuerzo o acomodo de forma que las estructuras respondan a ciertos patrones institucionales en respuesta a la contingencia, por ello los planes de capacitación que ha impulsado el Consejo son una tarea muy importante en este proceso de instalación antes de comenzar las fiscalizaciones.

Finalmente destacar que las relaciones de poder marcan fuertemente el deseo por posicionarse en el espacio institucional, principalmente por parte de los altos funcionarios, con el objeto de legitimar los recursos y acciones que lleva adelante el Consejo para desplegar su estrategia, vale decir, aumentar el conocimiento de la ley 20.285, y que también son mencionadas por el grupo "otros" cuando llaman a contar con una cultura de transparencia, a su vez los gestores lo refuerzan al hacer mención del "empoderamiento ciudadano y eficiencia por parte de los organismos del Estado". Para todo lo anterior, se hace mención a cómo distintos grupos de interés pueden marcar el destino que tome la política, ya sea, por medio del control de recursos o la negociación, dado que la política puede alterar ciertos intereses presentes en cierto grupo. 


\section{Acciones que encauzaron al Consejo a concretar valor público}

El Consejo, como se desprende de los testimonios, al dotar a sus procesos de una orientación ciudadana, materializan el concepto de valor público, haciendo que sus labores sean concordantes con el cumpliendo de sus objetivos, pues su trabajo pretendería generar una mejora en la calidad de atención, permitiendo entregar una experiencia de servicio más concordante con las verdaderas necesidades que tienen las personas.

Este propósito por marcar una orientación ciudadana, hizo que tanto en la misión como en los objetivos estratégicos que proclama el Consejo, se coloque especial énfasis en la capacidad de respuesta del Consejo al cliente, lo que se ve reflejado en las palabras de un entrevistado: "conocer bien a nuestro cliente, determinar cuál era su requisito, introducir esos requisitos en nuestras cadenas productivas, que tenemos 5 negocios, y después monitorearnos permanentemente respecto a la satisfacción usuaria". Tareas como las descritas anteriormente, son parte de la manera o forma en la cual un organismo puede utilizar el empoderamiento de funciones.

Así también, el trabajo en red se hace indispensable cuando se requiere de otros para lograr los objetivos, y en este aspecto, el valor público contribuiría a generar esas conexiones. En este caso, el Consejo estableció alianzas con otros organismos públicos de modo de conseguir apoyo, aprendizaje y facilitar procesos, en especial cuando se está recién comenzando a instalar un organismo, como comenta un entrevistado: "logramos acuerdos con la dirección del Servicio Civil, incluso iniciamos las conversaciones con ellos antes de que nosotros estuviéramos ratificados por el Senado. De modo que una vez de que el Consejo existiera como tal, pudiésemos inmediatamente llamar a concurso y publicar en los diarios que correspondiese, los llamados a concursos".

Otra forma de afianzar relaciones de parte del Consejo con otros actores de la sociedad civil fue por medio de reuniones. De este modo, el Consejo va sumando respaldo político y ciudadano a sus labores y atribuciones como órgano mandante y garante del derecho de acceso a la información.

Las relaciones de poder marcan el proceso de implementación, debido a los conflictos surgidos para alcanzar cierto posicionamiento en el espacio interorganizacional. Conflictos como el que desencadenó que una gran campaña de medios para dar a conocer al Consejo, tuviese que ser acotada en tema de recursos, para también dar prioridad a la construcción institucional del organismo. Esto y otros incon- venientes comentados en capítulos anteriores, hacen que según las declaraciones dadas por los grupos de entrevistados, la implementación se haya concentrado en un comienzo en los actores más políticos principalmente, considerando de este modo, muy importante la opinión de los actores centrales que participaron en la formulación o diseño de la PAIP, por eso la relevancia en cumplir con los objetivos y fines que se encuentran en la ley 20.285.

\section{Discusión}

El análisis de los testimonios de los entrevistados deja ver los factores que predominaron durante el proceso de instalación de la PAIP por parte del Consejo. Estos fueron: las relaciones de poder, búsqueda de la racionalidad y ajuste organizacional, y, de modo más marginal discreción burocrática y adaptación; y liderazgo y competencia.

Estos resultados son explicados principalmente porque las tensiones sobre el control de los recursos que se destinarían al Consejo, coparon la preocupación al interior de este organismo. Por otra parte, las disputas por el espacio institucional con la Comisión Asesora Ministerial para la Probidad y la Transparencia, fue percibida por los entrevistados como una factor que marcó el destino que fue tomando el proceso de instalación, aquellos eventos hacen que las relaciones entre los diferentes grupos de interés hayan sido difíciles en un comienzo, porque todo política pública trastoca valores que son importantes para un segmento de la sociedad.

Otro aspecto importante que afirman los entrevistados, tiene relación con la administración del Portal de Transparencia, pues los testimonios revelan que conseguir la tutoría de este portal está directamente relacionada con la capacidad del Consejo para la Transparencia.

Dado lo anterior, se aprecia también a partir de los relatos, incluso en mayor medida en el grupo de gestores públicos, la preocupación por establecer procesos en torno a resultados, aspectos muy propios de un enfoque gerencial, que por consiguiente conduce naturalmente a dirigir la atención al valor que se desea transmitir a la sociedad, por ello la racionalidad se hace presente fuertemente, así lo afirman los enunciados, de ese modo se logra que los ejecutores se comporten según el estatuto o mandato político.

Los ajustes a la política se hicieron presentes durante el proceso de instalación, toda vez, que con el objeto de obtener resultados a partir de la construcción de procesos, se hizo uso por parte del Consejo, 
de herramientas de gestión. El uso de la cadena de valor y la creación de tareas como la capacitación como una forma de hacer frente a problemas que la instalación de la PAIP había arrojado en otros organismos públicos.

Las relaciones de poder se plasma en esta política, porque primero es una política de reforma del Estado que impacta en el que hacer interno de los servicios, en otras palabras, genera un nuevo modo en que el Estado va a trabajar, por ende, al ser algo con una mirada a largo plazo, es razonable esperar que los las relaciones de poder tengan cierta preminencia frente a otros factores, pues trastoca valores y dinámicas de trabajo perpetuadas por años dentro del sistema público, y en particular, el mundo político se expone en mayor medida al juicio ciudadano.

¿Qué hace diferente los resultados de este estudio a los de Hasenfeld \& Brock (1991) y los de Olavarría (2014)? Primeramente la metodología de cada estudio es diferente, mientras que el de Hasenfeld \& Brock (1991) está basado en la percepción de los escritores de esos 37 estudios analizados, el de Olavarría (2014), se realizó a partir del testimonio de 67 entrevistas. Y este trabajo se basa en el relato de 11 entrevistas.

Pero más allá de la metodología ocupada, la principal diferencia de este trabajo con el estudio de Hasenfeld \& Brock (1991), tiene relación con la características de las políticas analizadas. Si bien, para ambos trabajos las relaciones de poder son una fuerza predominante en el proceso de instalación de la política, antes hay que señalar que, para el primer estudio las políticas analizadas corresponden a políticas sociales, estas se caracterizan por tener procesos altamente conflictivos, porque corresponderían a una especie de política redistributiva según señalan Ripley y Franklin (1982, citado en Hasenfeld y Brock, 1991), además del conflicto, la aplicación es menos aceptada por la generalidad de las personas.

En cambio, en este estudio, se analizó una política (Política de Acceso a la Información Pública), que no posee las características de una política redistributiva, porque no trata de criterios que dan acceso a ventajas, y de este modo, las personas pueden beneficiarse de alguna cobertura social, como es en el caso de las políticas de seguridad social. La PAIP es, dentro de ese esquema, una política constitutiva (Lowi, 1972), dado que el beneficio afecta indistintamente a todos en la sociedad e este modo, la acción pública define normas o reglas acerca del poder, ejemplo de ello es la introducción de reformas institucionales o administrativas, afectando al ciudadano de modo menos directo que otras políti- cas públicas.

Que en ambos trabajos las relaciones de poder hayan sido una fuerza predominante, es producto principalmente del conflicto y la tensión en la cual estuvo expuesta la política en el transcurso de la implementación. En el caso de Hasenfeld \& Brock, (1991), las políticas sociales al ser redistributivas, el organismo ejecutor influyó en el proceso de aplicación, en especial los intereses de la coalición dominante (el grupo que controla los recursos entró en conflicto con los instrumentos de la política). Algo similar ocurre en la implementación de la PAIP, la tensión por los recursos expresada en las entrevistas, fue un modo de controlar el proceso por parte del gobierno, o insertar límites a las acciones que pudiesen emprender el Consejo, por eso la tensión se produce en los dos primeros años de funcionamiento del Consejo para la Transparencia, así también la dilatación por entregar la administración del Portal de Transparencia al Consejo también significó retrasar una verdadera consolidación de funciones para este organismo en términos de operación.

Otro aspecto que sumó para que las relaciones de poder fueran tan patentes en este proceso de implementación, fue el continuo esfuerzo por parte del Consejo por consolidarse en el espacio interorganizacional, ya que este estuvo permeado por conflictos que dieron cuenta de que en esta relación institucional existía una idea de contrapeso de poder.

Si bien, para el estudio de las políticas sociales, otra fuerza predominante fue la adaptación burocrática, que en realidad fue otra forma de manifestación de las relaciones de poder, por el contrario, en el caso de este estudio la siguiente fuerza de gran influencia según los entrevistados fue la búsqueda de racionalidad, por el carácter técnico, principalmente a nivel micro, en la labor de los directivos de segundo nivel. Esto es concordante con los resultados del estudio de Olavarría (2014:24), toda vez que esta política es un peldaño más dentro del contexto de la Política de Modernización de la Gestión del Estado. Por lo tanto, requiere de un conocimiento experto porque los cambios a introducir se "concentraban dentro de la administración pública", por eso el uso de la planificación estratégica de forma que el resultado sea lo más acorde a una ejecución racional, de este modo en base a la dotación de recursos disponibles, se diseñaron los modelos de gestión y procesos necesarios para el cumplimiento de objetivos trazados según el mandato político, o la idea de valor público, que quería imprimir el Consejo en la sociedad. Así también se destaca en los testimonios, la orientación a resultados de todos sus procesos, este enfoque permite que las acciones y procesos se alineen de mejor forma a un valor que 
la misma organización declara por medio de la misión, visión y objetivos estratégicos, de este modo, promueve al interior como al exterior una propuesta de valor público a la sociedad y quiénes sean sus usuarios.

La siguiente fuerza predominante en este trabajo es el ajuste organizacional de la política. Por ello el rol de la institucionalidad del Consejo es influyente a la hora de estructurar las direcciones y unidades de trabajo más habituales en respuesta a las atribuciones que le dotaba la ley 20.285 al Consejo para la Transparencia. Esto se fue generando de modo gradual. Algo similar ocurre en el Estudio de implementación de la Política de Reforma y Modernización de la GEstión Pública, donde también el ajuste organizacional estuvo presente en los resultados del análisis, dado que los objetivos de esta política consisten en ir institucionalizando los mecanismos creados en el transcurso de instalación de la Política de Reforma y Modernización de la GEstión Pública.

La discreción burocrática, presente en el estudio de Hasenfeld \& Brock (1991) como un factor importante, parece no ser una fuerza muy influyente en el proceso de implementación de la PAIP, según plantean los entrevistados. Esto se debe porque primó el ajuste organizacional a la hora de hacer frente a la cultura funcionaria, que no tenía la práctica de entregar información del Estado al ciudadano, ya sea por un tema valórico o por ver en esta implementación una mayor carga de trabajo, indirectamente esta política está afectando prácticas laborales que estaban instauradas por años dentro de la administración del Estado, forzando tanto a los políticos como a los gestores públicos al cambio.

Por otra parte, en el trabajo de Olavarría (2014), se presenta el liderazgo y competencia como una fuerza preponderante en el proceso de instalación de la Política de Reforma y Modernización de la GEstión Pública, en cambio, para el caso de la implementación de la Política de Acceso a la Información Pública, según los relatos, se encuentra como la última fuerza de las cinco que analiza este enfoque. Las razones, según las evidencias de los grupos de entrevistados, pueden ser la estructura del Consejo, que si bien es el organismo garante del derecho y promotor de la PAIP, el hecho de que el Consejo se constituya como un gobierno corporativo, en donde la figura del Consejero es de carácter rotativo, y el durar 18 meses en su calidad de presidente, en donde sus funciones no son muy distintas que la de los otros consejeros, claro si, en caso de fallos su votación en dirimente, por lo tanto el presidente tiene pocas atribuciones ejecutivas. En cambio, la imagen del Director general, quien sí puede llegar a tener una mayor duración en su cargo, dota al orga- nismo según testimonio de los mismos funcionarios del Consejo, de un liderazgo interno de gestión propiamente, porque en él recae la responsabilidad de administrar el Consejo y su funcionamiento, y con ello tiene varias facultades: puede contratar, puede desvincular, puede asignar recursos, pero siempre tiene que informar del desempeño de esta corporación, al menos una vez al mes, a los Consejeros, además, la figura del Director no está afecta directamente a los ciclos políticos.

Que las relaciones de poder, la búsqueda de racionalidad y el ajuste organizacional hayan sido las principales fuerzas que influyeron o explican principalmente la implementación de la PAIP, parece ser consistente con las dinámicas de una política con un enfoque de top down en términos macro a nivel político, pero a medida que se va instalando la política, esta se va ajustando a un nivel micro en base al contexto inter-organizacional que obliga a los gestores públicos (a nivel de calle), quienes tienen más cercanía con el problema, a adecuar la política acorde a los requerimientos ciudadanos.

Un enfoque como este, ayudará a los políticos, gestores y funcionarios del Estado a analizar el proceso e indagar cuales serían las razones por las que la gente no manifiesta mayor dominio sobre el tema, o no ve un aporte sustancial en su vida diaria. Y por tanto, hacer los cambios necesarios para ir empoderando a la ciudadanía sobre el derecho, abriéndole espacios para que accedan a otros derechos, por medio del uso y aplicación de políticas como la que en este trabajo se analiza, generando un fortalecimiento de la democracia cada vez que la sociedad haga uso de la ley 20.285, y vaya asumiendo el dominio de este derecho como parte importante en el desarrollo de su vida en sociedad.

\section{Conclusiones}

Los factores predominantes en la implementación de la PAIP por parte del Consejo son: relaciones de poder $(30,19 \%)$, búsqueda de la racionalidad $(25,13 \%)$ y ajuste organizacional de la política $(19,06 \%)$, los que representan en su conjunto un gran porcentaje, $74,38 \%$, de los testimonios entregados en las entrevistas realizadas.

Por lo tanto, desde el punto de vista de las fuerzas predominantes, permite dar cuenta que los temas críticos en la instalación de la PAIP por parte del Consejo, han sido la obtención de recursos presupuestarios, la administración del Portal de Transparencia, el posicionamiento del Consejo para la Transparencia dentro de espacio interorganizacional y la cultura funcionara cristalizada 
por años en donde la solicitud de información como un bien público no se encontraba arraigada como un derecho ciudadano y por tanto exigible. Así también desde un aspecto más jurídico, se vislumbra un conflicto que tienen relación con la manera en cómo se nombra a los consejeros, que da cuenta de una falta de transparencia y que en un futuro cercano podría traer complicaciones a la hora de proyectar un verdadero respaldo por parte de la sociedad a las declaraciones que emanen de este organismo, afectando así su posicionamiento y respaldo ciudadano.

En cuanto al tema presupuestario, también se deja ver un conflicto futuro, pues este tema hace que el Consejo se homologue a cualquier otra subsecretaria de gobierno, dado que, se trata de un organismo con autonomía propia pero vinculada financieramente al Gobierno de turno y supervisado por la Contraloría, por lo tanto, se da una supervisión y control de modo indirecto a las acciones que emprenda el Consejo y por consiguiente una falta de autonomía funcional de este organismo.

En cuanto al posicionamiento, también en el trascurso del análisis de las entrevistas, se infieren dificultades en cuanto a la institucionalidad del Consejo, justamente por ese nivel de mixtura: por un lado es un órgano colectivo de cuatro miembros (consejeros), con un presidente que dura 18 meses, el cual no es un jefe de servicio y, por lo tanto, no tiene ninguna atribución orgánica. Además por otro lado, existe la figura de un Director General, elegido por concurso público y que actúa como representante legal del Consejo para la Transparencia. Esto genera que exista al interior del organismo una distinción marcada por estos dos roles, en donde el Presidente junto a los Consejeros se centran fundamentalmente en resolver casos, y el Director General junto a su equipo administrativo se hacen cargo del funcionamiento cotidiano del Consejo, esto último justifica el carácter racional que ha tenido el proceso de implementación de la PAIP, pues este cuerpo de directivos de segundo nivel operacionalizaron la política para darle cuerpo y estructura a lo que planteaba la LAIP que el Consejo debía llevar adelante.

También se reconoce que existe de parte de todos los entrevistados una idea común, de lo que se quiere alcanzar en tema de resultados, es el aumento del conocimiento de la ley 20.285 y por consiguiente el empoderamiento ciudadano que a la larga obliga a los organismos públicos a ser más eficientes en sus tareas con la sociedad, y de modo interno, el Consejo declara continuamente su orientación al ciudadano en el enfoque de todos sus procesos, con el propósito de acercar sus labores y no perder de vista al usuario final de esta PAIP. Lo anterior, son aspectos representativos de un enfoque de valor público, porque pretenden crear valor a partir de las transacciones individuales con los ciudadanos, garantizando sus derechos, satisfaciendo sus demandas y mejorando continuamente la calidad del servicio entregado.

A partir del análisis y discusión presentados en este trabajo, se puede entender con mayor claridad el por qué una política puede generar mayor o menor rechazo, justamente por el cómo se ha llevado la implementación de esta mediante el reconocimiento de factores driving forces domains, los cuales impactarán de modo diferente en el curso de instalación de la política, dada las características de la política en cuestión, dependiendo del área, materia e interés que trate, de este modo, los efectos y dinámicas serán distintos de una política a otra, dependiendo del área impactada por la política.

En relación a la labor de los gerentes públicos en la implementación de la PAIP, el predominio de las relaciones de poder, señala cuán importante es que este actor sepa relacionarse, tanto dentro del escenario político como en el técnico, pues las políticas requieren en el mayor de los casos un respaldo, o sea, ser políticamente sustentable y eso se logra negociando con los diferentes grupos de interés que se encuentran principalmente en la fase de formulación y diseño de la política.

De igual manera, el predominio de la racionalidad refleja cuán impregnado se encuentra, en la actual administración pública de Chile, el concepto de administración por resultados, con el objeto de hacer de la implementación algo técnicamente factible. En parte por el impacto que tuvo la nueva gestión pública en América Latina según se plantea en Ramírez (2009), caló hondamente en cómo diseñar procesos en términos de eficiencia y eficacia, con el objeto de hacer un buen uso de los recursos públicos, pero también hay que tener en cuenta que el exceso por hacer de este enfoque el único capaz de responder a las necesidades estatales, puede hacer olvidar quien es el destinatario último de la política, el ciudadano y la sociedad en general.

Por último, si bien se reconoce que es importante que los gestores conozcan de herramientas de gestión como son la planificación estratégica, el uso de indicadores, el cuadro de mando integral, estos juegan un rol importante en temas de ajuste de la política, para alinear a la organización hacia fines estratégicos, el sólo hecho de hacer uso de estos recursos no asegura el éxito de la implementación de la política, necesariamente se requiere que se logre lo que Moore (1998) mediante el uso del triángulo estratégico establece, que la declaración de valor que promueva el servicio sea sustentable políticamente. Para ello, es importante un trabajo 
con el entorno inter-organizacional con el propósito de posicionarse en el espacio institucional, logrando aquello, también hay que asegurar que sea técnicamente factible, o sea, contar con los recursos tanto tecnológicos como humanos, y primero que todo que la propuesta de valor sea sustantivamente valiosa, que la sociedad reconozca que con ello da respuesta a problemas que son relevantes para los ciudadanos o usuarios.

\section{Referencias}

Bardin, L. (2002). Análsis de contenido. Akal, S.A, Madrid.

Elmore, R. F. (1987). Instruments and strategy in public policy. Policy Studies Review, 7(1):174186.

Hasenfeld, Y. y Brock, T. (1991). Implementation of Social Policy Revisited. Administration $\&$ Society, 22(4):451-479.

Hill, M. y Hupe, P. (2002). Implementing Public Policy. SAGE Publications Ltd, London.

Horner, L. y Hutton, W. (2011). Public Value, Deliberative Democracy and the Role of Public Managers. En Public Value Theory $\&$ Practice, pp. 112-126. Palgrave Macmillan, New York.

Klijn, E. H. (2007). Networks an Inter - Organizational Management: Challenging, Steering, Evaluation, and the role of public actors in public management. En Lynn, L. E. y Pollitt, C., editores, The Orford Handbook of Public Management, pp. 257-281. Orford University Press, New York.

Lawrence, P. R. y Lorsch, J. W. (1967). Organization and Environment. Harvard Business School, Boston.

Levin, M. A. y Ferman, B. (1985). The Political Hand: Policy Implementation and Youth Employment Programs. Pergamon Press, New York.
Lowi, T. J. (1972). Four Systems of Policy, Politics, and Choice. Public Administration Review, $32(4): 298-310$.

Moore, M. (1995). Creating Public Value: Strategic Management in government. Harvard University Press, Massachussets.

Moore, M. H. (1998). La estratégia organizativa en el sector público. En Gestión Estratégica y creación de valor público, pp. 101-162. Ediciones Paidós, Buenos Aires.

Olavarría Gambi, M. (2012). La institucionalización y gestión estratégica del acceso a la información y la transparencia activa en Chile. Banco Interamericano de Desarrollo y Consejo para la Transparencia, Chile.

Olavarría-Gambi, M. (2014). Policy Implementation: Lessons from the Chilean Policy on Public Management Modernization. University of Santiago, Chile, Santiago, Chile.

Ramírez, M. (2009). Las reformas del Estado y la administración pública en América Latina y los intentos de aplicación del New Public Management. Estudios Políticos, 34(1):115-141.

Sabatier, P. A. (1987). Knowledge, policy-oriented learning and policy change, An Advocacy Coalition Framework. Knowledge, 8(4):649-692.

Weatherley, R. y Michael, L. (1977). Street-Level Bureaucrats and Institutional Innovation: Implementing Special Education Reform in Massachusetts. Harvard Educational Review, 47(2):171197.

Yin, R. (1994). Investigación sobre estudio de casos: Diseño Y Métodos. Applied Social Research Methods Series, 5(2). 